\title{
Detection of RNAP-DNA complexes using solid state nanopores
}

\author{
C. Raillon, P. Granjon, M. Graf and A. Radenovic
}

\begin{abstract}
Transcription is the first step in gene expression where DNA is copied into RNA. It is extensively studied at the bulk level especially the regulation mechanism, which in cancerous cells is impaired. We were interested in studying $\mathbf{E}$. coli RNAP enzyme at the single-molecule level for its functional as well as molecular motor properties. With nanopore sensing, we were able to observe RNA polymerase-DNA complexes translocate through nanopores and able to distinguish between individual complexes and bare RNA polymerase. We were also able to observe orientation of RNA polymerase in the nanopore whether flow or electric field predominates. The complexity of the signals from the protein-DNA complexes experiment motivated us to develop level detection software. This software is based on a change detection method called the CUSUM algorithm. OpenNanpore software was designed to analyze in details current blockages in nanopore signals with very little prior knowledge on the signal. With this work one can separate events according to their number of levels and study those subpopulations separately.
\end{abstract}

\section{INTRODUCTION}

In this paper, we summarize recent findings on the power of nanopore-based sensing to detect and identify specific proteins or DNA-protein complexes based on the shape of its corresponding ionic current translocation traces $[1,2]$. Nanopore-based analysis is an innovative technique used for a wide range of applications such as molecule length measurement, single-molecule dynamics or sequencing [4-6].

In the past decade, many single-molecule techniques have been used for a wide variety of biological systems: proteins, nucleic acids or complexes of the two. Most of what we know today about the transcription reaction was learnt from bulk studies, in which one measures the average signal issued from a very large number of molecules. In recent year, however, researchers have started measuring fundamental properties using single-molecule experiments. For example, by attaching a single molecular motor such as RNAP to a bead, researchers have been able to probe properties like step size, speed and force using optical tweezers [7-10]. Here, we

*Research supported by the European Research Council (grant no. 259398, ProABEL: Nanopore integrated nanoelectrodes for biomolecular manipulation and design.

C.R. Author is with Laboratory of Nanoscale Biology, Institute of Bioengineering, School of Engineering, EPFL, 1015 Lausanne, Switzerland (e-mail: camille.raillon@epfl.ch).

P.G. Author is with Grenoble Image Speech Signal Automatics Laboratory, Grenoble Institute of Technology, Grenoble, France (pierre.granjon@gipsa-lab.grenoble-inp.fr)

M.G: Author is with Laboratory of Nanoscale Biology, Institute of Bioengineering, School of Engineering, EPFL, 1015 Lausanne, Switzerland (e-mail: michel.graf@epfl.ch).

A.R. Author is with Laboratory of Nanoscale Biology, Institute of Bioengineering, School of Engineering, EPFL, 1015 Lausanne, Switzerland (corresponding author to provide phone: 041-21-6937371; fax: 041-216937380; e-mail: aleksandra.radenovic@epfl.ch). show how nanopore sensing and nanopore based force spectroscopy can be used to study RNAP-DNA interaction at the single-molecule level.

\section{DETECTION OF RNAP-DNA COMPLEXES USING SOLID STATE NANOPORES}

\section{A. Solid state nanopore fabrication}

For single-molecule applications such as nanopore sensing, the nanopore chip should satisfy several requirements. The membrane should contain one pore only, and the pore size should be comparable to the DNA diameter ( $2 \mathrm{~nm}$ for dsDNA) so that DNA passes through the pore in a linear fashion and the ion flux is significantly reduced when DNA molecule translocates.

The pore geometry needs to be well defined and reproducible. In addition, the pore surface should be hydrophilic to facilitate the microfluidic cell filling with solution and it should be neutral or positively charged to avoid DNA repulsion. The membrane has to be strong enough to withstand experiments for several hours but thin enough to make a nanopore in it. The chip should also tolerate salt concentrations ranging from $0.1-2 \mathrm{M} \mathrm{KCl}$ and different $\mathrm{pH}$ conditions $(2-9 \mathrm{pH})$. The starting substrate, for nanopore fabrication, is a double-sided polished silicon wafer with a triple stack of insulating layers, $20 \mathrm{~nm}$ of low-stress silicon nitride $\left(\mathrm{Si}_{\mathrm{x}} \mathrm{N}_{\mathrm{y}}\right), 100 \mathrm{~nm}$ of silicon dioxide $\left(\mathrm{SiO}_{2}\right)$ and $100 \mathrm{~nm}$ of $\mathrm{Si}_{\mathrm{x}} \mathrm{N}_{\mathrm{y}}$. The supporting silicon and insulating layers on the backside are dry and wet etched to form a $50 \times 50 \mu \mathrm{m}^{2}$ large, $220 \mathrm{~nm}$ thick membrane. Then electron beam lithography (EBL) is used to define a small square $(500 \times 500$ $\mathrm{nm}^{2}$ ) in the center of the $\mathrm{Si}_{\mathrm{x}} \mathrm{N}_{\mathrm{y}}$ membrane. This region is then thinned to less than $20 \mathrm{~nm}$ using dry and wet etching.

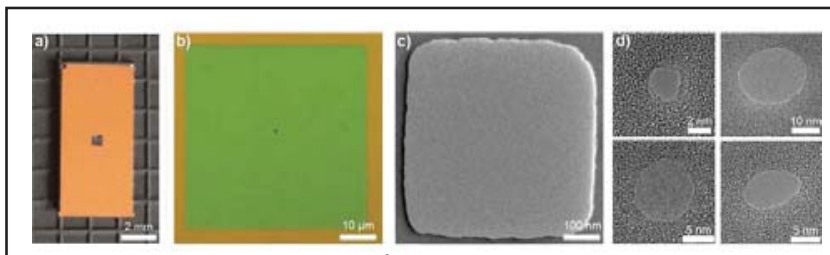

Figure 1. a) $4 \times 8 \mathrm{~mm}^{2}$ silicon chip, $\mathrm{KOH}$ groove is visible in the center of the chip b) Optical image of a 50 x $50 \mu \mathrm{m}^{2} \mathrm{Si}_{\mathrm{x}} \mathrm{N}_{\mathrm{y}}$. membrane (in green) in the center of the chip shown in a). This $220 \mathrm{~nm}$ thick membrane is composed of three stacked layers $(20 \mathrm{~nm}$ low-stress $\mathrm{Si}_{\mathrm{x}} \mathrm{N}_{\mathrm{y}}, 100 \mathrm{~nm} \mathrm{SiO}$ and $100 \mathrm{~nm}$ low-stress $\mathrm{Si}_{\mathrm{x}} \mathrm{N}_{\mathrm{y}}$.) and is created by standard processes of photolithography, dry and wet etching. The small black dot in the center of the membrane corresponds to the thinned region c) TEM image of the thinned region; fabricated by electron beam lithography and etched down to a thickness of $20 \mathrm{~nm}$. d) TEM images of several nanopore sizes. Taken from [3]. 
This allows the creation of a very thin membrane, mechanically stable because made of $\mathrm{Si}_{\mathrm{x}} \mathrm{N}_{\mathrm{y}}$. That region is thin enough to drill a nanometer-sized hole with a highly focused electron beam. In this microfabrication process, we obtain 72 chips per wafer one such chip is shown in Figure 1. The tool used to drill nanoscale holes is typically a Transmission Electron Microscope (TEM). We use a Philips/FEI CM300 to make the nanopores. Nanopores form due to localized heating that fluidizes matter, and surface tension that creates geometry-dependent contraction or expansion [12]. The optimal parameters for nanopore drilling are following: an accelerating voltage of $200 \mathrm{kV}$ and an extraction voltage of $4.2 \mathrm{kV}$ using condenser lens 3 and spot size $5(\sim 10 \mathrm{~nm})$ at a magnification of $220000 \mathrm{X}$. In TEM mode, the beam is focused with the intensity knob for about 1 min. Sometimes it can take up to several minutes to obtain a nanopore; this mostly depends on the chip cleanliness. There are several ways to clean a nanopore chip from hydrocarbon contamination, we often use $\mathrm{O}_{2}$ plasma.

The problem with this contamination is that it can migrate into the electron beam and be immobilized [13]. We were originally drilling nanopores at an accelerating voltage of 300 $\mathrm{kV}$ but, in order to limit hydrocarbon contamination we have lowered it to $200 \mathrm{kV}$ and we have used the cold trap; which condenses all vapors and so limits contamination at the nanopore drilling site. It is also best to wait that drift stabilizes before starting the drilling process, and $30 \mathrm{~min}$ is usually enough. The use of TEM as a nanopore fabrication tool allows for the variation of nanopore sizes. Figure $1 \mathrm{~d}$ ) shows four nanopores ranging from 2 to $20 \mathrm{~nm}$. This is extremely important since it allows tuning of the pore size, one of the prerequisites for recognition of protein-DNA complexes [3].

\section{B. Measurement conditions and setup}

Once the pore is fabricated, it is put into a microfluidic cell that is filled with an ionic solution Figure 2 a). To optimize translocation signal, the size of the pore is matched to the size of the protein, DNA or Protein DNA complex. In our case pores slightly bigger than RNAP-DNA (around 20 $\mathrm{nm}$ ) complex gave best results.

To get an ionic flow, the pore needs to be hydrophilic, to do so we store the chips in degassed and filtered 1:1 $\mathrm{ddH}_{2} \mathrm{O}: \mathrm{EtOH}$ solution until use. This straightforward step yields highly reproducible wettability of nanopores. Two other solutions are to use $\mathrm{O}_{2}$ plasma or to deposit aluminum hydroxide $\left(\mathrm{Al}_{2} \mathrm{O}_{3}\right)$ on the chip after pore drilling [13]. ALD (Atomic Layer Deposition) can also be used to precisely control the nanopore diameter. In our hands, the first simple solution of storing nanopores in a mixture of water and ethanol worked better than the $\mathrm{Al}_{2} \mathrm{O}_{3}$ deposition.

Transparent microfluidic cells allow for fast detection, hence removal, of large air bubbles. An Axopatch 200B (Molecular Devices, Inc. Sunnyvale, CA) is used to amplify the ionic current through the nanopore. The Axopatch is a low noise patch clamp amplifier widely used in nanopore setups. The $\mathrm{Ag} / \mathrm{AgCl}$ electrodes are immersed in the buffer solution immersed in the reservoirs on each side of the nanopore and connected to the Axopatch preamplifier.

The microfluidic cell and the Axopatch preamplifier are mounted on a damping breadboard (Thorlabs, NJ) and closed in a Faraday cage.

The sample is introduced into the cis side of the nanopore, and a voltage is applied across the membrane In the absence of a flow, the DNA will face two different regimes, one regime that is diffusion limited (far away from the nanopore) and one regime that depends on the pore diameter, its length and the applied voltage (closer to the nanopore. Signals are filtered at $10 \mathrm{kHz}$ using the low-pass Bessel filter built-in the Axopatch and sampled at $100 \mathrm{kHz}$ using a National Instrument PXI-4461 DAQ card. This allows for low-noise measurement.

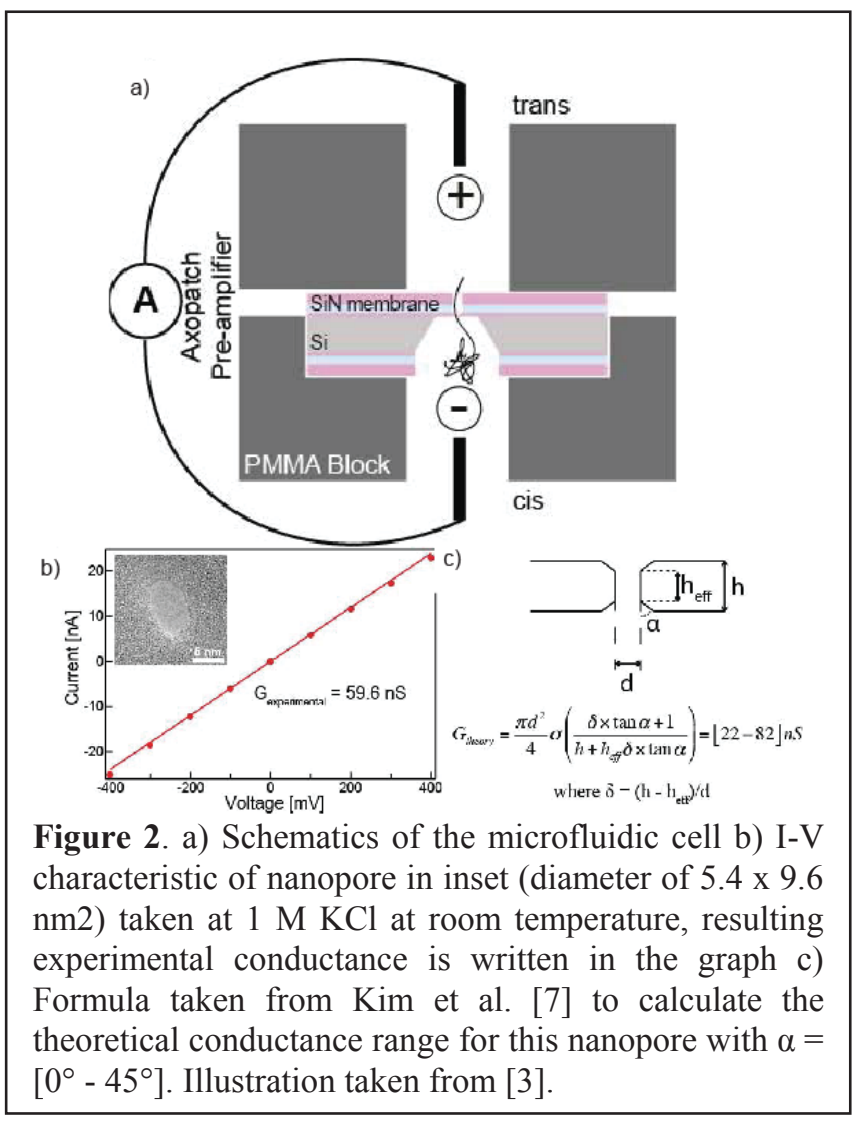

To allow for discrimination of events above the noise level, a custom made LabVIEW program only records data when the current signal peak depth is above $4 \times$ IRMS and the event longer than $50 \mu \mathrm{s}$, which makes files lighter and easier to analyze. The applied voltage can be controlled directly on this interface. This same voltage can be swept to establish the I-V characteristic of the nanopore before starting the experiment Figure 2 b) and c) [2, 3].

\section{RESUlts}

The higher the voltage, the bigger the capture radius [14]. In the experiments on bare RNAP and RNAP-DNA 
complexes, we tried to keep the applied voltage as low as possible $(50 \mathrm{mV})$ in order to minimize the electrophoretic force and maximize the dwell times for each event. This proved to be successful in most experiments and allowed us to visualize orientation of bare RNA whether force or flow predominates as well as detect individual protein-DNA complexes [2, 3]. Typical ionic current events from experiment with RNAP-DNA complexes with RNAP halted at one end of the DNA template are shown in Figure 3.

In our recent publications [2, 3] we described the appropriate volume-based approach using previous knowledge on RNAP, its dipole, its charge and its atomic structure. For the first time, we have shown that at low bias voltage:

1) RNAP translocates in different measureable orientations whether it is the RNAP dipole or the drag force that predominates.

2) using a volume-based approach we have shown that we could distinguish between bare RNAP and single RNAPDNA complexes using nanopore sensing.at higher voltages:

3) RNAP subunits could break off the core enzyme in measurable subunits

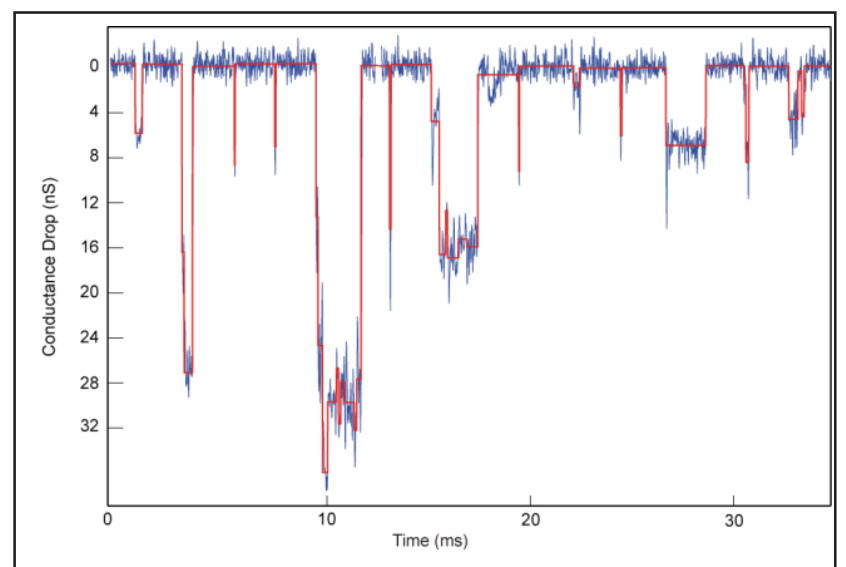

Figure 3. Concatenated events from experiment with RNAP-DNA complexes with RNAP halted at one end of the DNA template. Example of a current trace (in blue) after addition of RNAP-DNA complexes to the $16 \times 19 \mathrm{~nm}$ pore in $1 \mathrm{M} \mathrm{KCl}$ solution with $50 \mathrm{mV}$ applied. In red the fit obtained using the cumulative sum (CUSUM) algorithm. Illustration taken from [1].

\section{OPENNANOPORE SOFTWARE}

The complexity of our nanopore signals tailored the needs for a level-detection software that we developed [2]. This software is based on the cumulative sum (CUSUM) algorithm that does fast and automated detection of events and their levels even when the signal to noise ratio is close to 1 . A major advantage of this software is that events can be classified according to their number of levels and so it is easy to make statistics on sub-populations. This new method has demonstrated promising results on complex signals such as protein-DNA complexes. This new software was critical to demonstrate the small difference of levels depending on the orientation of the translocating molecule $[2,3]$.

As mentioned above, in a typical nanopore experiment a constant voltage is applied between the two sides of the pore and the current passing through the pore is measured. Thanks to the electrical potential, charged molecules are driven through the pore (if their size allows it). During this translocation process, the molecules provoke a current blockade in the pore, which gives a characteristic current drop motif. This current-drop motif, which is generated during molecule transition through the pore, is referred to as event.

The software uses two key concepts the CUSUM algorithm and a recursive lo pass filtering. With this software we can compute event dwell times and conductance drops for each level inside each event. All events are classified as one, two or multi-level event. Statistics can also be performed directly with the OpenNanopore Statistics. The GUI (Graphical User Interface) is extremely intuitive each point in the scatter plot can be selected, and the event is then displayed at the bottom. Dwell time and current blockage histograms are displayed on each side of the scatter plot. Both histograms are level histograms; this means each level corresponds to one count in the histogram. Such tools allow the user to select a sub-population of events based on the number of levels in each event and do statistical studies on each population separately. We have first validated OpenNanopore software on prototypical data of $\lambda$ DNA molecules translocating a nanopore.

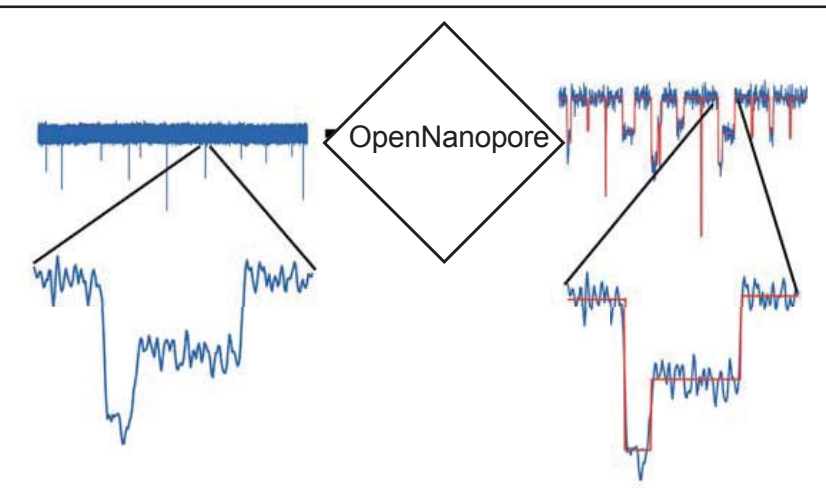

Figure 4. Depiction of the OpenNanopore's tasks :

Level fitting and creating database of fitted events.

In the current blockage histogram displayed in Figure 5 from our recent publication [2], it is easy to distinguish two sub-populations of events, the one-level events (in green) and the two-level events (in blue and red). What stems out is that one-level events are centered around two different current blockages: 0.2 and $0.4 \mathrm{nA}$ but the majority is around the 0.2 $\mathrm{nA}$ peak whereas, for two-level events, the quantity of $0.2 \mathrm{nA}$ levels is equal to the quantity of $0.4 \mathrm{nA}$ levels. This shows the statistical repartition of $\lambda$ DNA molecules translocating a nanopore one-level events are mostly events where the DNA molecule translocates in a linear and unfolded fashion and two-level events are events where the DNA molecule translocates partially unfolded $(0.2 \mathrm{nA})$ and partially folded 
(0.4 nA). Such a statistical study can be used further in experiments with proteins and biocomplexes where the aim is to distinguish between different sizes of molecules and their orientation while translocating the nanopore $[2,3]$.

OpenNanopore software can be downloaded from http://lben.epfl.ch/page-79460.html.

\section{CONCLUSION}

Nanopore sensing is a versatile technique; which can also be integrated, in more complex platforms such as optical tweezers, to do force measurement on molecular motors for example. In 2006 Keyser et al. [15] published the first experiment of optical trapping force measurement of DNA translocating through a nanopore giving a direct measurement of the electrical force exerted on DNA during translocation. This recent concept of coupling nanopores to optical tweezers opened up a variety of new single molecule studies for biomolecules [16, 17]. This technique could be used to further explore molecular mechanics of transcription, to study forces applied by RNAP to DNA during transcription and give a more detailed view of the kinetic of transcription.

Compared to current single-molecule experiments done on RNAP [8-10] nanopore unfolding can remove structure by shearing rather than pulling. It can also open structures strictly sequentially as they pass through the nanopore. Such an experimental configuration more accurately simulates "natural" biological processes such as transcription [3].

\section{ACKNOWLEDGMENT}

Authors thank to D.T.L. Alexander for providing training and technical assistance with the transmission electron microscope. Nanopore fabrication was carried out in part in at the EPFL Center for Micro/Nanotechnology (CMI). Thanks to K. Lister (CMI) for technical support with electron-beam lithography.

\section{REFERENCES}

[1] C. Raillon, P. Cousin, F. Traversi, E. Garcia-Cordero, N. Hernandez, and A. Radenovic, "Nanopore detection of single molecule RNAP-DNA transcription complex," Nano letters, vol. 12, pp. 1157-64, Mar 142012.

[2] [C. Raillon, P. Granjon, M. Graf, L. J. Steinbock, and A. Radenovic, "Fast and automatic processing of multi-level events in nanopore translocation experiments," Nanoscale, vol. 4, pp. 4916-24, Aug 212012.

[3] C. Raillon, "Nanopore sensing of single molecules application to RNAP-DNA complexes, fabrication of graphene-nanopore devices and translocation algorithm analysis," $\mathrm{PhD}$ thesis, Laboratoire de biologie à l'échelle nanométrique École polytechnique fédérale de Lausanne EPFL, , Lausanne, 2012.

[4] John J. Kasianowicz, E. Brandin, D. Branton, and David W. Deamer, "Characterization of individual polynucleotide molecules using a membrane channel," PNAS, vol. 93, pp. 13770-13773, November 26, 1996 1996.

[5] M. Akeson, D. Branton, J. J. Kasianowicz, E. Brandin, and D. W. Deamer, "Microsecond Time-Scale Discrimination Among Polycytidylic Acid, Polyadenylic Acid, and Polyuridylic Acid as Homopolymers or as Segments Within Single RNA Molecules," Biophys. J., vol. 77, pp. 32273233, December 1, 19991999.

[6] A. Meller, L. Nivon, E. Brandin, J. Golovchenko, and D. Branton, "Rapid nanopore discrimination between single polynucleotide molecules," Proceedings of the National Academy of Sciences of the United States of America, vol. 97, pp. 1079-1084, February 1, 20002000.

[7] M. J. Kim, M. Wanunu, D. C. Bell, and A. Meller, "Rapid fabrication of uniformly sized nanopores and nanopore arrays for parallel DNA analysis," Advanced Materials, vol. 18, pp. 3149-+, Dec 42006.

[8] M. D. Wang, M. J. Schnitzer, H. Yin, R. Landick, J. Gelles, and S. M. Block, "Force and velocity measured for single molecules of RNA polymerase," Science, vol. 282, pp. 902-907, Oct 301998.

[9] R. J. Davenport, G. J. L. Wuite, R. Landick, and C. Bustamante, "Single-molecule study of transcriptional pausing and arrest by E-coli RNA polymerase," Science, vol. 287, pp. 2497-2500, Mar 312000.

[10] J. W. Shaevitz, E. A. Abbondanzieri, R. Landick, and S. M. Block, "Backtracking by single RNA polymerase molecules observed at near-basepair resolution," Nature, vol. 426, pp. 684-687, Dec 112003.

[11] K. M. Herbert, A. La Porta, B. J. Wong, R. A. Mooney, K. C. Neuman, R. Landick, and S. M. Block, "Sequence-resolved detection of pausing by single RNA polymerase molecules," Cell, vol. 125, pp. 1083-94, Jun 162006.

[12] A. J. Storm, J. H. Chen, X. S. Ling, H. W. Zandbergen, and C. Dekker, "Fabrication of solid-state nanopores with single-nanometre precision," Nature materials, vol. 2, pp. 537-40, Aug 2003.

[13] A. Radenovic, E. Trepagnier, R. Csencsits, K. H. Downing, and J. Liphardt, "Fabrication of $10 \mathrm{~nm}$ diameter hydrocarbon nanopores," Applied Physics Letters, vol. 93, Nov 32008.

[14] M. Wanunu, W. Morrison, Y. Rabin, A. Y. Grosberg, and A. Meller, "Electrostatic focusing of unlabelled DNA into nanoscale pores using a salt gradient," Nature Nanotechnology, vol. 5, pp. 160-165, Feb 2010 .

[15] U. F. Keyser, B. N. Koeleman, S. Van Dorp, D. Krapf, R. M. M. Smeets, S. G. Lemay, N. H. Dekker, and C. Dekker, "Direct force measurements on DNA in a solid-state nanopore," Nature Physics, vol. 2, pp. 473-477, Jul 2006.

[16] E. H. Trepagnier, A. Radenovic, D. Sivak, P. Geissler, and J. Liphardt, "Controlling DNA capture and propagation through artificial nanopores," Nano letters, vol. 7, pp. 2824-2830, Sep 2007.

[17] [A. Spiering, S. Getfert, A. Sischka, P. Reimann, and D. Anselmetti, "Nanopore Translocation Dynamics of a Single DNA-Bound Protein," Nano letters, vol. 11, pp. 2978-2982, Jul 2011. 